\title{
DEMONSTRATION OF MUTATION
}

\section{DEVELOPMENT AND VIRULENCE CHANGE IN REFERENCE CANDIDA STRAINS EXPOSED TO CASPOFUNGIN}

\author{
Nurnehir Baltaci ${ }^{1,2}$, Ayse Kalkanci ${ }^{1}$ \\ ${ }^{1}$ Gazi University, School of Medicine, Department of Medical Microbiology, Ankara, Turkey \\ ${ }^{2}$ Afyonkarahisar Health Sciences University, Faculty of Pharmacy, Department of Pharmaceutical Microbiology, \\ Afyonkarahisar, Turkey.
}

Address for Correspondence: Assist. Prof. Nurnehir Baltaci, E-mail: nurnehirgazi@gmail.com Received: 20.04.2021; Accepted: 20.05.2021; Available Online Date: 27.05.2021

(C) Copyright 2021 by Dokuz Eylül University, Institute of Health Sciences - Available online at https://dergipark.org.tr/en/pub/jbachs

Cite this article as: Baltaci N, Kalkanci A. Demonstration of Mutation Development and Virulence Change in Reference Candida Strains Exposed to Caspofungin. J Basic Clin Health Sci 2021; 2: 115-123.

\begin{abstract}
Purpose: Echinocandin resistance is causing problems in the treatment. FKS gene mutations were detected on the genomes of the resistant Candida strains exposed to echinocandins. The aim of this study was to compare the resistance of reference Candida strains exposed to caspofungin and to investigate whether there was a difference in virulence factors between exposed and non-exposed with caspofungin.
\end{abstract}

Methods: Caspofungin susceptible reference strains, exposed to caspofungin during their incubation in caspofungin containing agar plates at concentrations of $16-0.03 \mu \mathrm{g} / \mathrm{ml}$. MIC values of caspofungin were determined all strains. FKS gene regions of the caspofungin exposed strains were sequenced in order to reveal the difference between the reference strains. Relationship between caspofungin exposure and virulence properties were investigated in vivo and in vitro methodologies. The in vivo virulence study on the mini-experimental animal model Galleria mellonella (wax moth) was performed.

Results: MIC values of the caspofungin exposed strains were found to be increased as one/two fold dilution when compared to the sensitive reference strains. The result of FKS gene sequence no mutation is detected in the reference strains, while a point mutation in caspofungin exposed C. glabrata strains were detected. No significant difference was found in virulence properties of reference strains and caspofungin exposed strains.

Conclusions: In conclusion, it was understood that the fact that sensitive strains were exposed to caspofungin even at low concentrations caused an increase in MIC, and that virulence and drug resistance should be evaluated independently.

Keywords: Candida, Caspofungin, FKS mutation, Galleria mellonella, virulence

\section{INTRODUCTION}

Candida species are the fourth most common cause of bloodstream infections (1). Invasive fungal infections due to Candida spp. are important causes of morbidity and mortality, especially in immunocompromised patients. Antifungals used in the treatment of these patients are limited. Echinocandin class are a newer, selective and semisynthetic antifungal group than other antifungal 
Table 1. DNA primers used in the study

\begin{tabular}{lll}
\hline Function and primers & Primer sequence $\left(\mathbf{5}^{\prime} \rightarrow \mathbf{3}^{\prime}\right)$ & $\underline{\text { References }}$ \\
\hline $\begin{array}{l}\text { Candida universal FKS1HS1 } \\
\text { Candida universal FKS1 HS1 }\end{array}$ & AAT GGG CTG GTG CTC AAC AT & $(11)$ \\
Candida universal FKS1 HS2F & CCT TCA ATT TCA GAT GGA ACT TGA TG \\
Candida universal HS2R & AAG ATT GGT GCT GGT ATG GG & $(12)$ \\
$\begin{array}{l}\text { C. albicans fks1 hot spot } 1 \\
\text { C. albicans fks1 hot spot } 1\end{array}$ & TAA TGG TGC TTG CCA ATG AG \\
$\begin{array}{l}\text { C. albicans fks1 hot spot } 2 \\
\text { C. albicans fks1 hot spot } 2\end{array}$ & TTCACCATTACATCTCAT & $(11)$ \\
Candida glabrata FKS1 & AAGATTGGTGCTGGTATGGG & $(13)$ \\
Candida glabrata FKS1 & ACCTCTTTCAATCAATTCTTGAACAAC & $(13)$ \\
\hline
\end{tabular}

drugs. This class of antifungals acts by targeting the enzyme $\beta-(1,3)-D$-glucan synthase in the fungal cell

wall. Resistance to antifungals of the echinocandin class develops due to mutations in the "hot spot" regions of the FKS gene encoding the glucan synthase enzyme (2). Clinical factors supporting resistance include prophylactic use of echinocandins and empirical echinocandin treatment. In recent years, studies emphasizing the importance of resistance to antifungals of the echinocandin class have been increasing $(3,4)$. It was reported that echinocandin resistance was reported worldwide, ranging from $0.1-3.5 \%$ for Candida glabrata, $C$. albicans and $C$. parapsilosis (4). No caspofungin resistance has been reported in our country. The relationship between exposure to caspofungin or the development of resistance and the pathogenesis of diseases caused by these strains and their virulence characteristics has not yet been fully elucidated. The number of studies on this subject is limited (5).

The aim of this study is to convert the reference strains of $C$. albicans, C. parapsilosis and C. glabrata into mutants in the presence of caspofungin and to analyse the phenotypic and genotypic aspects and reveal the virulence characteristics of these strains.

\section{METHODS}

\section{Comparison experiments with caspofungin}

Solid media containing caspofungin were prepared, and an agar dilution-based method was applied. Candida albicans ATCC 10231, Candida glabrata
ATCC MYA-2950 and Candida parapsilosis ATCC 22019, which were sensitive and had not previously encountered caspofungin, were used as reference strains in the study. For exposure with an echinocandin, Sabouraud Dextrose Agar (SDA) plates were prepared, containing caspofungin (Sigma, USA) at decreasing concentrations starting from $16 \mu \mathrm{g} / \mathrm{mL}$ to $0.03 \mu \mathrm{g} / \mathrm{mL}$ (6). A total of $10 \mu \mathrm{L}$ of the yeast suspension was taken from the yeast suspension at a concentration of $10^{6}$ and spread to the SDA plates containing caspofungin by a swab. Plates were incubated at $37^{\circ} \mathrm{C}$ for 48 hours to allow growth. Simultaneously, drug-free media were used as controls. Colonies that grew under caspofungin were accepted as "caspofungin resistant mutant". The concentration of caspofungin was noted $(6,7)$. The following formula was used to calculate mutation frequencies (8). Both mutant and parent strains were stored at $-80^{\circ} \mathrm{C}$.

Mutation frequency $=$ Colony number $(\mathrm{CFU} / \mathrm{mL})$ / Initial inoculum (CFU/mL)

\section{Antifungal sensitivity test}

MIC values of reference strains that had not previously encountered antifungals and strains exposed to caspofungin were determined. For this purpose, reference microdilution methods, M27-A3 and M27-S4, standardized for yeasts by the Clinical Laboratory Standards Institute (CLSI) were used $(9,10)$. MIC values for amphotericin B, fluconazole, voriconazole, caspofungin, and itraconazole were evaluated twice at 24 hours and 48 hours. The 
Table 2: MIC results of reference strains and strains exposed to caspofungin and CLSI M27 S3/M27 S4 standards (9,10).

\begin{tabular}{|c|c|c|c|c|c|c|c|c|c|c|c|c|c|}
\hline \multirow{2}{*}{ Antifungals } & \multicolumn{2}{|c|}{ CLSI M27-S3 } & \multicolumn{3}{|c|}{ CLSI M27 S4 } & \multirow[b]{2}{*}{$\mathbf{R}$} & \multirow[b]{2}{*}{ Strains } & \multicolumn{2}{|c|}{ MIC $(\mu \mathrm{g} / \mathrm{ml})$} & \multirow[b]{2}{*}{ Antifungals } & \multicolumn{2}{|c|}{$\mathrm{MIC}(\mu \mathrm{g} / \mathrm{ml})$} & \multirow{2}{*}{$\begin{array}{l}\text { After the exposed to } \\
\text { echinocandins }\end{array}$} \\
\hline & $\mathrm{s}$ & SDD/I & $\mathbf{R}$ & $\mathrm{s}$ & SDD/I & & & $\begin{array}{l}24 \\
\text { hours }\end{array}$ & $\begin{array}{l}48 \\
\text { hours }\end{array}$ & & $\begin{array}{l}24 \\
\text { hours }\end{array}$ & $\begin{array}{l}48 \\
\text { hours }\end{array}$ & \\
\hline $\begin{array}{l}\text { Amphotericin } \\
\text { B }\end{array}$ & - & - & - & - & - & - & \multirow{5}{*}{$\begin{array}{l}\text { C. albicans ATCC } \\
10231\end{array}$} & 0.03 & 0.06 & $\begin{array}{l}\text { Amphotericin } \\
\text { B }\end{array}$ & 0.06 & 0.06 & \multirow{5}{*}{$\begin{array}{l}\text { C. albicans } \\
\text { mutant }\end{array}$} \\
\hline Fluconazole & - & - & - & $\leq 2$ & 4 & $\geq 8$ & & 0.5 & 0.5 & Fluconazole & 2 & 2 & \\
\hline Voriconazole & - & - & - & $\leq 0.12$ & $\begin{array}{l}0.25- \\
0.5\end{array}$ & $\geq 1$ & & 0.125 & 0.125 & Voriconazole & 0.25 & 0.25 & \\
\hline Caspofungin & - & - & - & $\leq 0.25$ & 0.5 & $\geq 1$ & & 0.03 & 0.03 & Caspofungin & 0.03 & 0.03 & \\
\hline Itraconazole & - & - & - & - & - & - & & 0.03 & 0.06 & Itraconazole & 0.06 & 0.125 & \\
\hline $\begin{array}{l}\text { Amphotericin } \\
\text { B }\end{array}$ & - & - & - & - & - & - & \multirow{5}{*}{$\begin{array}{l}\text { C. parapsilosis } \\
\text { ATCC } 22019\end{array}$} & 0.03 & 0.03 & $\begin{array}{l}\text { Amphotericin } \\
\text { B }\end{array}$ & 0.03 & 0.03 & \multirow{5}{*}{$\begin{array}{l}\text { C. parapsilosis } \\
\text { mutant }\end{array}$} \\
\hline Fluconazole & - & - & - & $\leq 2$ & 4 & $\geq 8$ & & 2 & 2 & Fluconazole & 1 & 1 & \\
\hline Voriconazole & - & - & - & $\leq 0.12$ & $\begin{array}{l}0.25- \\
0.5\end{array}$ & $\geq 1$ & & 0.125 & 0.125 & Voriconazole & 0.125 & 0.5 & \\
\hline Caspofungin & - & - & - & $\leq 2$ & 4 & $\geq 8$ & & 0.125 & 0.125 & Caspofungin & 0.25 & 0.5 & \\
\hline Itraconazole & - & - & - & - & - & - & & 0.03 & 0.03 & Itraconazole & 0.03 & 0.03 & \\
\hline $\begin{array}{l}\text { Amphotericin } \\
\text { B }\end{array}$ & - & - & - & - & - & - & & 0.03 & 0.03 & $\begin{array}{l}\text { Amphotericin } \\
\text { B }\end{array}$ & 0.03 & 0.03 & \multirow{5}{*}{$\begin{array}{l}\text { C. glabrata } \\
\text { mutant }\end{array}$} \\
\hline Fluconazole & - & - & - & - & $\leq 32$ & $\geq 64$ & & 8 & 8 & Fluconazole & 16 & 16 & \\
\hline Voriconazole & - & - & - & - & - & - & $\begin{array}{l}\text { C. glabrata ATCC } \\
\text { MYA } 2950\end{array}$ & 0.125 & 0.125 & Voriconazole & 0.125 & 0.125 & \\
\hline Caspofungin & - & - & - & $\leq 0.12$ & 0.25 & 0.5 & & 0.03 & 0.03 & Caspofungin & 0.125 & 0.125 & \\
\hline Itraconazole & - & - & - & - & - & - & & 0.06 & 0.06 & Itraconazole & 0.03 & 0.03 & \\
\hline
\end{tabular}

Abbreviations: S: Sensitive, SDD/I: Susceptible Dose Dependent/Intermediate, R: Resistant

MIC values obtained from C. albicans ATCC 10231, C. parapsilosis ATCC 22019, and C. glabrata ATCC MYA-2950 reference strains were 0.03, 0.125, and 0.03 $\mu \mathrm{g} / \mathrm{mL}$, respectively. While MIC values of C. albicans were unchanged after exposure to caspofungin, they increased to $0.25 \mu \mathrm{g} / \mathrm{mL}$ at $24 \mathrm{hours}$ and $0.5 \mu \mathrm{g} / \mathrm{mL}$ at 48 hours for C. parapsilosis strain and to $0.125 \mu \mathrm{g} / \mathrm{mL}$ for C. glabrata strain.

sensitivity tests were repeated four times to test the persistence of sensitivity in mutant strains using RPMI medium prepared at different times.

\section{DNA isolation}

DNA isolation from the strains was performed according to the protocol using "The High Pure PCR Template Preparation Kit" (Roche, Switzerland). The total amount of DNA was measured on the spectrophotometer (NanoDrop, USA). The prepared DNAs were stored in a stock solution at $-80^{\circ} \mathrm{C}$. The DNA amounts were remeasured and checked prior to sequence analysis.

\section{Gradient polymerase chain reaction}

During the Polymerase Chain Reaction (PCR) process, "Gradient PCR" was applied to find the appropriate primer binding temperature (annealing). All primers used in this study were synthesized at Sentegen (Ankara, Turkey). T100 Thermal Cycler (Bio-RAD, USA) was used for "Gradient PCR".

\section{DNA sequence analysis}

Sequence analysis of FKS1 and FKS2 "Hot Spot" regions was performed to determine whether there was a mutation in the FKS gene region of the strains exposed to caspofungin. Predefined primer sequences were used in sequence analysis (11-14). The primer sequences used are presented in Table 1. Candida universal primers were used for $C$. parapsilosis that is not listed.

For the sequence analysis, the "Beckman Coulter Ampure XP" kit (Beckman Coulter, USA) was used for DNA purification. The DNA product was pre-treated using the "BigDye Terminator Cycle Sequencing Kit" (Applied Biosystems, USA). Capillary electrophoresis was performed by loading the products obtained after cycle sequencing to "Applied Biosystems 3130 Genetic Analyzer" (Applied Biosystems, USA) after purification with "ZR-96 DNA Sequencing Clean-up Kit" (Zymo Research Corp, USA). The obtained electropherograms were analysed with the "SnapGene 4.1.9" (Chicago, USA) program. The sequences were then converted to the "FASTA" format and compared with the sequences of the strains used in the study using the Basic Local Alignment Search Tool program at NCBI GenBank, and mutation analysis was performed. 
Table 3. Virulence characteristics of reference strains and strains exposed to caspofungin.

\begin{tabular}{|c|c|c|c|c|c|c|c|c|c|c|}
\hline \multirow[b]{2}{*}{ Strain Names } & \multirow[b]{2}{*}{$\begin{array}{l}\text { Caspofungi } \\
\mathrm{n} \mathrm{MIC} 48 \mathrm{~h}- \\
(\mu \mathrm{g} / \mathrm{ml})\end{array}$} & \multirow[b]{2}{*}{$\begin{array}{l}\text { Mutation in } \\
\text { the FKS } \\
\text { region }\end{array}$} & \multicolumn{7}{|c|}{ In Vitro Virulence Assays Results } & \multirow[b]{2}{*}{$\begin{array}{l}\text { Average } \\
\text { Larval } \\
\text { Mortality (\%) }\end{array}$} \\
\hline & & & $\begin{array}{l}\text { Phospholi } \\
\text { pase } \\
\text { Activity }\end{array}$ & $\begin{array}{l}\text { Esteras } \\
\text { e } \\
\text { Activity }\end{array}$ & $\begin{array}{l}\text { SAP } \\
\text { Activit } \\
y\end{array}$ & $\begin{array}{l}\text { SAP } \\
\text { Diametre } \\
(\mathrm{mm} / \mathrm{mm})\end{array}$ & $\begin{array}{l}\text { Biofil } \\
\text { m } \\
\text { Activit } \\
\text { y } \S\end{array}$ & $\begin{array}{l}\text { Adhesi } \\
\text { on } \\
\text { Activit } \\
\text { y (\%) }\end{array}$ & $\begin{array}{l}\text { Hemolysis } \\
\text { Production }\end{array}$ & \\
\hline C. albicans ATCC 10231 & 0.03 & - & + & + & + & $1.4 / 0.6=2.3$ & - & $73 \%$ & $\alpha$-hemolysin & $67 \%$ \\
\hline C. albicans Mutant & 0.03 & - & - & - & + & $0.6 / 0.4=1.5$ & - & $55 \%$ & Non hemolytic & $71 \%$ \\
\hline C. parapsilosis ATCC 22019 & 0.125 & - & - & - & + & $0.8 / 0.5=1.6$ & - & $79 \%$ & $\alpha$-hemolysin & $64 \%$ \\
\hline C. parapsilosis Mutant & 0.5 & - & - & - & + & $1.3 / 0.6=2.16$ & ++ & $87 \%$ & $\alpha$-hemolysin & $68 \%$ \\
\hline C. glabrata ATCC MYA-2950 & 0.03 & - & - & - & - & - & - & $78 \%$ & Non hemolytic & $58 \%$ \\
\hline C. glabrata Mutant & 0.125 & $\begin{array}{l}\text { position } 203 \\
\text { of the FKS1 } \\
\text { gene region }\end{array}$ & - & - & - & - & - & $85 \%$ & Non hemolytic & $80 \%$ \\
\hline
\end{tabular}

$\S$ Strains were classified into four groups according to their abilities to form biofilms. The four groups were as follows: (-) no biofilm producers, $(+)$ weak biofilm producers, $(++)$ moderate biofilm producers, $(+++)$ strong biofilm producer. The strong biofilm-producing strain was not identified in this study.

\section{Virulence assays}

Changes in virulence characteristics and phenotypes of ATCC strains exposed to caspofungin were investigated. For this purpose, adhesion assay, biofilm assay, haemolytic activity assay, secretory aspartyl proteinase (SAP) activity, phospholipase and esterase production were investigated in vitro. Adhesion assay, biofilm assay, haemolytic activity assay, SAP activity and phospholipase production were studied as reported by Vieira de Melo et al. (15). The percentage of adhesion to buccal epithelium was calculated in the adhesion assay. For the biofilm assay, the tube adherence method was used. For the haemolytic activity assay, sheep blood agar medium was used as modified to contain 3\% SDA. For SAP activity, bovine serum albumin-containing medium was used. For phospholipase activity, a medium containing sterile egg yolk was used. For esterase formation, a medium containing peptone and tween 80 was used.

All experiments were repeated twice. Pseudomonas aeruginosa ATCC 27853, Staphylococcus aureus ATCC 29213, and Streptococcus epidermidis ATCC 12228 were used as the control group in the experiments.

\section{Galleria mellonella in vivo virulence assay}

Galleria mellonella (wax moth) was grown in a dark laboratory environment at $28^{\circ} \mathrm{C} \pm 2^{\circ} \mathrm{C}, 60 \pm 5 \%$ relative humidity. The prepared medium (100 g) contained $22 \mathrm{~g}$ wheat flour, $22 \mathrm{~g}$ wheat bran, $11 \mathrm{~g}$ milk powder, $5.5 \mathrm{~g}$ dry yeast, $17 \mathrm{~g}$ wax, $11 \mathrm{~mL}$ glycerol, and $11 \mathrm{~mL}$ honey mixture. 10 randomly selected white larvae, approximately $300 \mathrm{mg}$, were distributed to sterile petri dishes. Suspensions containing $10^{6}$ CFU/mL yeast cells prepared from ATCC strains and caspofungin-exposed strains were prepared in sterile saline. A total of $10 \mu \mathrm{L}$ yeast suspension was applied directly inside the posterior left leg of each larvae with a "Hamilton" injector. While making the infection model, larvae which were not treated as control group were separated. The larvae infected with yeast strains were incubated at $30^{\circ} \mathrm{C}$ to note the number of live larvae daily. With the number of live larvae, "Kaplan Meier" (survival) curves were plotted (16).

\section{Statistical analysis}

In order to compare the virulence of caspofunginexposed strains with standard strains, independent samples t-test (SPSS 22.0 [Statistical Program for Social Sciences, Chicago, USA]) was used. Independent samples t-test was preferred in the study because it is the most common method to investigate the difference between two main mass sample means. 


\section{RESULTS}

\section{Results of comparison with caspofungin}

For comparison with caspofungin, 15 separate consecutive passages were performed on SDA medium containing caspofungin at decreasing concentrations from $16 \mu \mathrm{g} / \mathrm{mL}$ to $0.03 \mu \mathrm{g} / \mathrm{mL}$. There was no growth in the media with caspofungin amount between $16-2 \mu \mathrm{g} / \mathrm{mL}$. The amount of caspofungin was reduced in narrow intervals after $2 \mu \mathrm{g} / \mathrm{mL}$. C. albicans ATCC 10231 strain began to grow on medium containing $0.48 \mu \mathrm{g} / \mathrm{mL}$ caspofungin, C. glabrata ATCC MYA-2950 strain on medium containing 0.18 $\mu \mathrm{g} / \mathrm{mL}$ caspofungin, C. parapsilosis ATCC 22019 strain on medium containing $1.48 \mu \mathrm{g} / \mathrm{mL}$ caspofungin . These values were recorded as "mutant formation values." Colonies grown on caspofungin-containing medium were counted and divided into the initial amount of inoculum and thus, "mutation frequencies" were determined. Mutation frequency was $1.5 \times 10^{-5}$ for $C$. albicans, $1 \times 10^{-4}$ for C. glabrata, and $1 \times 10^{-4}$ for C. parapsilosis. These experiments were repeated three times. The viability of colonies grown under caspofungin was determined by performing passages on SDA plates without caspofungin.

MIC values obtained from reference and caspofungin exposure strains and amphotericin B, fluconazole, voriconazole, caspofungin, and itraconazole MICs and CLSI breakpoints were presented in Table 2 .

Two-way sequence analysis of $C$. albicans ATCC 10231 strain, C. parapsilosis ATCC 22019 strain and those exposed to caspofungin was performed with primers given in Table 1. No mutations were detected when the sequences obtained from the analysis were compared to gene bank data.

Two-way sequence analysis of the C. glabrata ATCC MYA-2950 strain and the 5th primer of the strain exposed to caspofungin was performed, and the presence of mutation was investigated. While there was no mutation in the reference strain, it was observed that thymine nucleotide $(T)$ was converted to cytosine nucleotide $(C)$ in the base at position 203 in the sequencing performed with the forward primer in the sequence analysis of the FKS1 gene region of the strain exposed to caspofungin (GenBank Accesion Number: MT079859).

C. albicans and C. parapsilosis strains could not be mutated and MIC values of caspofungin did not change. Mutations accompanied by increased MIC in C. glabrata strain were shown.

\section{Virulence Assays}

In the second part of the study, virulence characteristics of reference strains and strains exposed to caspofungin were investigated. The results of all virulence experiments are presented in Table 3.

C. albicans ATCC 10231 reference strain did not mutate after exposure to caspofungin, and its MIC value did not change. While the reference origin was positive for haemolysis, phospholipase and esterase, the colonies obtained after exposure to caspofungin were found to be negative for haemolysis, phospholipase and esterase. SAP activity zone diameter decreased after exposure to caspofungin. Biofilm was negative in $C$. albicans strain. In the adhesion assay, the percentage of adhesion decreased from $73 \%$ to $55 \%$ after exposure to caspofungin. The larval mortality of strains obtained after caspofungin increased from $67 \%$ to $71 \%$.

C. parapsilosis ATCC 22019 reference strain did not mutate after exposure to caspofungin but MIC value was increased by one dilution. Since MIC value was 0.25 and $0.5 \mu \mathrm{g} / \mathrm{mL}$, no phenotypic resistance was shown in this strain according to CLSI breakpoints. The SAP zone diameter of this strain increased, biofilm became positive, and adhesion percentage increased. Phospholipase, esterase, haemolysis and in vivo virulence properties remained unchanged.

C. glabrata ATCC MYA-2950 reference strain became mutant after exposure to caspofungin and its MIC value increased by two dilutions. Since MIC value was $0.125 \mu \mathrm{g} / \mathrm{mL}$, phenotypic resistance was not shown in this strain according to CLSI breakpoints. The adhesion percentages of this mutant strain increased from $78 \%$ to $85 \%$, and the mean in vivo mortality increased from $58 \%$ to $80 \%$. Phospholipase, esterase, SAP, biofilm, and haemolysis properties were not changed. Table 3 . Virulence characteristics of reference strains and strains exposed to caspofungin.

In our study, infection model in Galleria mellonella larvae was established as an in vivo virulence experiment. Deaths started 24 hours, and the highest mortality rate was reached at 192nd hour (8th day). The larvae with no treatment were used as the control group when making the infection model. KaplanMeier curves were plotted with the number of larvae infected with the strains used in the study and survived until the last day of the experiment (Figure 1). Since the differences in mortality rates between the reference strains and caspofungin-exposed 


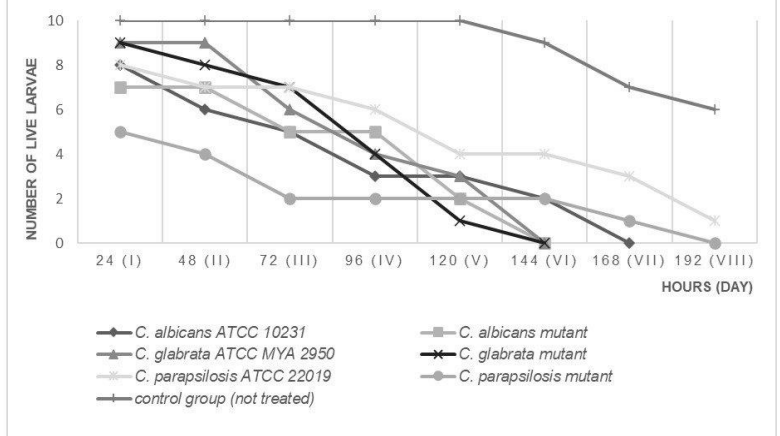

Figure 1: Survival curves of larvae

strains were not statistically significant $(p \geq 0.05)$, virulence was considered to be unaffected by exposure to caspofungin in the experimental larval model established.

\section{DISCUSSION}

In our study, reference Candida strains as Candida albicans ATCC 10231, Candida glabrata ATCC MYA2950, and Candida parapsilosis ATCC 22019, known to be susceptible to caspofungin, were exposed to caspofungin and were grown on drug-containing medium. Caspofungin MIC values of two strains other than $C$. albicans were shown to increase by one-two dilutions. Mutations were demonstrated in FKS gene regions responsible for echinocandin resistance in the C. glabrata strain. It was shown that in vitro virulence factors of mutant strains were partially changed compared to susceptible strains, whereas in vivo virulence characteristics of larvae were not different.

In the guidelines, echinocandins are recommended as the first treatment option of candidiasis in adult patients. Their use as the first treatment option in azole-resistant Candida spp. makes antifungals of the echinocandin class more valuable (17). Recent studies have reported the development of resistance due to exposure to echinocandin (4). There are case reports reporting resistance development after echinocandin use $(18,19)$. In our study, licensed laboratory strains whose characteristics were known were used. Of the strains, $C$. albicans began to grow on the medium containing $0.48 \mu \mathrm{g} / \mathrm{mL}, C$. parapsilosis on $1.48 \mu \mathrm{g} / \mathrm{mL}$, and C. glabrata on $0.18 \mu \mathrm{g} / \mathrm{mL}$. In the study of Chassot et al. 30 susceptible C. parapsilosis patient isolates were exposed to continuously increasing concentrations of caspofungin (7). Strains started to grow on medium containing $4.2 \mu \mathrm{g} / \mathrm{mL}$ caspofungin. Compared to the results of this study, it was found that the ATCC strains we used started to grow at a lower concentration. The reason for this difference was interpreted as the use of clinical strain by Chassot et al. (7). After the reference strains $C$. parapsilosis and C. glabrata started to grow under caspofungin in our study, caspofungin MIC values increased compared to baseline MIC values. However, this increase did not result in a "resistance". The caspofungin resistance breakpoint was reported as $8 \mu \mathrm{g} / \mathrm{mL}$ according to CLSI (20).

The presence of point mutation and deletion in the FKS region of C. glabrata strain exposed to caspofungin was shown in our study. These mutations were associated with increased MIC. The growth frequency under caspofungin was counted as 15 cells in $10^{6} \mathrm{C}$. albicans cells and C. parapsilosis, one cell in $10^{4} \mathrm{C}$. glabrata and cells. Since the term "exposure mutant" is used in the literature for "compatible" colonies that grow under drugs, this term is used in our study. However, no mutation in the $C$. albicans and C. parapsilosis genome were shown. Therefore, the above numbers, which are essentially determined as the "mutant frequency", should be considered as numbers of cells that adapt to reproduction under caspofungin.

Sequence analysis of the strain exposed to caspofungin obtained from C. glabrata ATCC MYA2950 showed a point mutation at position 203 of the FKS1 gene region. In another study, 77 of $C$. glabrata isolates isolated from 1380 patients with circulatory infection were found to be moderately susceptible and resistant to antifungals of the echinocandin class based on their MIC values. Several mutations were found in the FKS1 HS1 region in 15 of these and FKS2 HS1 region in 35 (21).

In our study, the virulence characteristics of strains exposed to caspofungin were examined. The hypothesis of our study was that; virulence characteristics of the strains exposed to echinocandins could be changed. C. albicans strain could not be mutated and the MIC value of caspofungin did not change. The virulence characteristics were partially altered after exposure to caspofungin. Phospholipase, esterase, and haemolysis properties were lost. SAP activity and adhesion percentages were also reduced. However, larval mortality was increased. Strains of the $C$. albicans species are rich in virulence factors (15). Various results were obtained in studies examining the relationship between echinocandin exposure and 
virulence in C. albicans strains. Ellepola et al. reported that there was no change in haemolysis properties of strains exposed to low-concentration caspofungin, amphotericin B, nystatin, ketoconazole and fluconazole for one hour in a study conducted with $C$. albicans strains obtained from oral cavities of healthy individuals and patients with various diseases (22). In a US study, it was shown that SAP5 expression increased within 1 hour after 7 hours of caspofungin exposure ranging from $4 \mu \mathrm{g} / \mathrm{mL}$ to 16 $\mu \mathrm{g} / \mathrm{mL}$ (23). Ellepola et al. conducted an adhesion assay after 1 hour exposure of $C$. albicans and $C$. dubliniensis to caspofungin. They showed that the adhesion of $C$. dubliniensis strains to the epithelium of the mouth decreased (24).

C. parapsilosis strain strain could not be mutated after exposure to caspofungin, but MIC value was increased. While SAP activity, biofilm production and adhesion percentage of this mutant strain was increased, phospholipase, esterase, haemolysis production, and larval mortality did not change. It has been reported that exposure to echinocandin reduces virulence in $C$. parapsilosis strains and evolves the strains (25).

Spontaneous mutations occur in FKS gene regions in C. glabrata strains. Exposure to caspofungin increases this mutation frequency (8). In our study, mutation was developed and MIC value was increased in the C. glabrata strain after exposure to caspofungin. The adhesion percentage of the mutant origin increased, and the average mortality in larvae increased. The ability of C. glabrata strains exposed to micafungin and caspofungin to produce biofilm of origin was reduced (26).

In our study, mortality of Galleria mellonella larvae was investigated. The mini experimental animal model was preferred instead of mammalian animals due to cost, ethical rules, and working time advantages. There was no difference between the mortalities of the strains in the larval infection model. Exposure to caspofungin did not alter virulence in vivo. Papp et al. reported that mutant C. parapsilosis strains showed low in vivo virulence (25). Kalkancı et al. developed larval models with resistant and susceptible fungal and bacterial strains. In that study, it was concluded that antibiotic and antifungal resistance did not alter the mortality of larval infection (16). In our study, the strains were not resistant to caspofungin. They were strains that were exposed to caspofungin and thus formed mutations in FKS regions. No virulence increase was detected after exposure to caspofungin in our strains.

\section{CONCLUSION}

In this study, it was observed that exposure to caspofungin caused FKS mutation in C. glabrata strains. The MIC values of caspofungin were increased in C. parapsilosis and C. glabrata strains. However, virulence did not change significantly in the mutant strain. In $C$. albicans strains which did not develop mutations and whose caspofungin MIC values did not change, some virulence characteristics were lost. In the light of these observations, it was concluded that exposure to caspofungin did not affect in vivo and in vitro virulence of Candida strains studied.

Conflict of Interest: No conflict of interest was declared by the authors.

Financial Disclosure: This work was supported by the TUBITAK (grant number SBAG 216S888) and Gazi University Projects of Scientific Investigation (grant number BAP 01/2017-05)

Ethics Statement: The authors confirm that the ethical policies of the journal, as noted on the journal's author guidelines page, have been adhered to. No ethical approval was required as the research in this article to microorganisms and wax moths.

Acknowledgements: This work was supported by the TUBITAK (grant number SBAG 216S888) and Gazi University Projects of Scientific Investigation (grant number BAP 01/2017-05). The authors wish to thank Dr. Alişan Baltaci from Yüksek Ihtisas University for helping with statistical analysis.

\section{REFERENCES}

1. Touil HF., Boucherit K, Boucherit-Otmani $Z$ et al. Optimum Inhibition of Amphotericin-B-Resistant Candida albicans Strain in Single-and MixedSpecies Biofilms by Candida and Non-Candida Terpenoids. Biomolecules. 2020;10:342.

2. Perlin DS. Mechanisms of echinocandin antifungal drug resistance. Ann New York Acad Sci 2015;1354:1-11.

3. Beyer R, Spettel K, Zeller I et al. Antifungal susceptibility of yeast bloodstream isolates collected during a 10-year period in Austria. Mycoses. 2019;62:357-367.

4. Pfaller MA, Diekema DJ, Turnidge JD et al. Twenty Years of the SENTRY Antifungal Surveillance Program: Results for Candida Species From 1997-2016. Open Forum Infect Dis 2019;6 Suppl 1:79-94.

5. Toutounji M, Tokajian S, Khalaf RA. Genotypic and phenotypic characterization of Candida albicans Lebanese hospital isolates resistant and 
sensitive to caspofungin. Fungal Genet Biol 2019;127:12-22.

6. Bordallo-Cardona MÁ, Escribano $\mathrm{P}$, de la Pedrosa EGG et al. In vitro exposure to increasing micafungin concentrations easily promotes echinocandin resistance in Candida glabrata isolates. Antimicrob Agents Ch. 2017; 61: e01542-16.

7. Chassot F, Venturini TP, Piasentin FB et al. Exploring the in vitro resistance of Candida parapsilosis to echinocandins. Mycopathologia. 2016;181:663-70.

8. Shields RK, Kline EG, Healey KR, et al. Spontaneous mutational frequency and FKS mutation rates vary by echinocandin agent against Candida glabrata. Antimicrob Agents $\mathrm{Ch}$. 2019;63:e01692-18.

9. Clinical and Laboratory Standards Institute. Reference method for broth dilution antifungal susceptibility testing of yeasts- Third Edition: M27-A3. NCCLS, Wayne, PA.2008.

10. Clinical and Laboratory Standards Institute. Reference method for broth dilution antifungal susceptibility testing of yeasts, Third Edition informational supplement M27-S3. NCCLS, Wayne, PA. 2008.

11. Lackner $M$, Tscherner $M$, Schaller $M$ et al. Positions and numbers of FKS mutations in Candida albicans selectively influence in vitro and in vivo susceptibilities to echinocandin treatment. Antimicrob Agents Ch. 2014;58: 362635.

12. Castanheira M, Woosley LN, Diekema DJ et al. Low prevalence of fks 1 hot spot 1 mutations in a worldwide collection of Candida strains. Antimicrob Agents Ch. 2010;54:2655-9.

13. Cheung $Y-Y$, Hui M. Effects of Echinocandins in combination with Nikkomycin $Z$ against invasive Candida albicans bloodstream isolates and the fks mutants. Antimicrob Agents Ch. 2017;61: e00619-17.

14. Dudiuk C, Gamarra S, Leonardeli F et al. Set of classical PCRs for detection of mutations in Candida glabrata FKS genes linked with echinocandin resistance. J Clin Microbiol. 2014; 52:2609-14.

15. de Melo AV, Zuza-Alves D, da Silva-Rocha W et al. Virulence factors of Candida spp. obtained from blood cultures of patients with candidemia attended at tertiary hospitals in Northeast Brazil. J Mycol Med. 2019;292:132-139.
16. Kalkancı A, Fouad A, Erdoğan $M$ et al. Bazı bakteri ve mantarların virülansının araştııılmasında Galleria mellonella'nın in vivo model olarak kullanılması. Mikrobiyol Bul. 2015; 49:366-76.

17. Pappas PG, Kauffman CA, Andes DR et al. Clinical practice guideline for the management of candidiasis: 2016 update by the Infectious Diseases Society of America. Clin Infect Dis. 2015;62:e1-50.

18. Grosset M, Desnos-Ollivier M, Godet $C$ et al. Recurrent episodes of Candidemia due to Candida glabrata, Candida tropicalis and Candida albicans with acquired echinocandin resistance. Med Mycol Case Rep. 2016;14:20-3.

19. Khan Z, Ahmad S, Mokaddas $E$ et al. Development of echinocandin resistance in Candida tropicalis following short-term exposure to caspofungin for empiric therapy. Antimicrob Agents Ch 2018; 62: AAC. 01926-17.

20. Chassot F, Venturini TP, Piasentin FB et al. Activity of antifungal agents alone and in combination against echinocandin-susceptible and-resistant Candida parapsilosis strains. Rev Iberoam Micol. 2019;36:44-7.

21. Pham CD, Iqbal N, Bolden $C B$ et al. The role of FKS mutations in C. glabrata: MIC values, echinocandin resistance and multidrug resistance. Antimicrob Agents Ch. 2014;58: 4690-6.

22. Ellepola AN, Khajah R, Jayatilake S et al. Impact of brief exposure to antifungal agents on the postantifungal effect and hemolysin activity of oral Candida albicans. J Appl Oral Sci. 2015;23:4128.

23. Ripeau J-S, Aumont F, Belhumeur $P$ et al. Effect of the echinocandin caspofungin on expression of Candida albicans secretory aspartyl proteinases and phospholipase in vitro. Antimicrob Agents Ch. 2002;46:3096-100.

24. Ellepola ANB, Chandy R, Khan ZU et al. Caspofungin-induced in-vitro post-antifungal effect and its impact on adhesion related traits of oral Candida dubliniensis and Candida albicans isolates. J Microbiol Immunol. 2016;60:160-7.

25. Papp C, Kocsis K, Tóth R et al. EchinocandinInduced Microevolution of Candida parapsilosis Influences Virulence and Abiotic Stress Tolerance. mSphere. 2018;3:e00547-18.

26. Rodrigues CF, Rodrigues ME, Henriques M. Susceptibility of Candida glabrata biofilms to 
echinocandins: Alterations in the matrix composition. Biofouling. 2018;34:569-7 